\title{
Debris-stimulated tumor growth: a Pandora's box?
}

\author{
Victoria M. Haak ${ }^{1,2,3}$ - Sui Huang ${ }^{4} \cdot$ Dipak Panigrahy $^{1,2}$ \\ Received: 25 August 2021 / Accepted: 1 October 2021 / Published online: 19 October 2021 \\ (c) The Author(s), under exclusive licence to Springer Science+Business Media, LLC, part of Springer Nature 2021
}

\begin{abstract}
Current cancer therapies aim at eradicating cancer cells from the body. However, killing cells generates cell "debris" which can promote tumor progression. Thus, therapy can be a double-edged sword. Specifically, injury and debris generated by cancer therapies, including chemotherapy, radiation, and surgery, may offset their benefit by promoting the secretion of protumorigenic factors (e.g., eicosanoid-driven cytokines) that stimulate regrowth and metastasis of surviving cells. The debris produced by cytotoxic cancer therapy can also contribute to a tumor microenvironment that promotes tumor progression and recurrence. Although not well understood, several molecular mechanisms have been implicated in debris-stimulated tumor growth that we review here, such as the involvement of extracellular vesicles, exosomal miR-194-5p, Bax, Bak, Smac, HMGB1, cytokines, and caspase-3. We discuss the cases of pancreatic and other cancer types where debris promotes postoperative tumor recurrence and metastasis, thus offering a new opportunity to prevent cancer progression intrinsically linked to treatment by stimulating resolution of tumor-promoting debris.
\end{abstract}

Keywords Debris · Tumor progression $\cdot$ Surgery $\cdot$ Radiation $\cdot$ Chemotherapy $\cdot$ Inflammation $\cdot$ Resolution of inflammation $\cdot$ Metastasis · Recurrence $\cdot$ Caspase-3 · Extracellular vesicles $\cdot$ Exosomal miR-194-5p · Bax $\cdot$ Bak $\cdot$ Smac $\cdot$ HMGB1 $\cdot$ Platelet activating factor $\cdot$ Specialized pro-resolving mediators

\section{Introduction}

The stimulation of tumor growth by dead cell debris is of high clinical relevance, independent of modality. Whether surgery, chemotherapy, radiation, anti-angiogenic, immunotherapy, or targeted therapy, interventions that seek the reduction of tumor burden involve tissue injury and cell death in the tumor bed. From numerous pre-clinical studies, the dead cell debris production has emerged as the material source of this paradoxical stimulation of tumor growth associated with treatment, which underlies the inherent limitation of cancer treatment. A variety of animal tumor models have demonstrated the growth-stimulating activity of injection of

Victoria M. Haak

vmhaak@buffalo.edu

1 Center for Vascular Biology Research, Beth Israel Deaconess Medical Center, Harvard Medical School, Boston, MA, USA

2 Department of Pathology, Beth Israel Deaconess Medical Center, Harvard Medical School, Boston, MA, USA

3 Jacobs School of Medicine and Biomedical Sciences, University at Buffalo, Buffalo, NY, USA

4 Institute for Systems Biology, Seattle, WA, USA tumor cell debris along with the tumor graft [1-3]. Established living cell tumors in mice can also produce cell debris in situ by systemic administration of chemotherapy, supporting the biological relevance of the debris-stimulated tumor growth [1]. These studies on debris generated by chemotherapy are consistent with previous observations of debris produced by radiation therapy by Laszlo Révész in 1956 who attributed the stimulation of tumor growth to diffusible factors that conditioned the tumor microenvironment (Révész effect) [4-6]. Thus, debris-stimulated tumor growth may present a novel target for preventing tumor growth and metastasis following treatment.

\section{What is "debris"?}

There are several mechanisms of cell death including apoptotic cell death, "autophagic cell" death, and necrotic cell death [1, 7-11]. As one of the hallmarks of cancer is impaired deregulation of apoptosis, therapies target promoting cell death $[12,13]$. Cellular "debris" is produced from this therapy strategy. "Debris" is the resulting dead cell fragments that have yet to be cleared by the immune system [1]. Cancer 
therapies, including radiation, chemotherapy, and surgery, trigger the release of soluble factors from dying tumor cells, which recruit immune cells to engulf the debris $[2,7,8]$. However, these therapies can be a double-edged sword as dying cells can also secrete pro-tumorigenic eicosanoids, cytokines, and other mediators between the dying tumor cells and the surviving tumor cells $[1-3,7,12,14-17]$. The goal in cancer therapy over the past century has been to induce cell death in order to halt and regress tumor growth. However, the induction of cell death (production of "debris") can also possess a "dark side" with oncogenic properties that can stimulate tumorigenesis $[9,11,18]$. For example, apoptotic cell death can exhibit oncogenic potential by stimulating immune and inflammatory responses as well as triggering tissue repair and regeneration leading to an "onco-regenerative niche" [10]. The rapid tumor growth of cancer can lead to apoptosis via hypoxia, and the continuous production of apoptotic debris promotes tumor growth via inflammation $[1,2,17]$. Therefore, even natural cell death in the tumor microenvironment can promote tumor repopulation as tumorigenesis can be accelerated with treatment-induced cell death $[1,15,19]$. After the generation of debris by cancer therapies, tumors can initiate a process of accelerated repopulation leading to tumor recurrence [19-21]. Elevated cleaved caspase-3, a pro-apoptotic effector molecule, is associated with shortened survival in several cancer types including gastric, ovarian, cervical, and colorectal cancers $[11,20]$. Importantly, high levels of spontaneous apoptotic cell death ("tumor cell debris") correlate with poor patient prognosis and/or survival in many cancer types including breast, head and neck (e.g., squamous cell carcinoma of the tongue and laryngeal), bladder, synovial sarcoma, liver, colorectal, ovarian, prostate, non-Hodgkin's lymphoma, and esophageal (as reviewed in [22]) [18, 23-34]. Elevated levels of tumor cell debris can lead to an aggressive pro-tumorigenic phenotype in pre-clinical models and in humans $[1-3,17,25,26,32-35]$. Thus, every attempt to kill cancer cells and cause tumor cell death is a doubleedged sword as the resulting unresolved debris stimulates the growth of surviving tumor cells $[1-3,9,11,14,15,18,21$, 36-39]. It is crucial to understand the mechanisms of debrisstimulated cancer progression in order to enhance current cancer therapies to stimulate the clearance and resolution of tumor cell debris $[21,40]$.

\section{Radiation-stimulated cancer}

Over the past century, cancer therapy has focused on killing cancer cells, from broad cytotoxic therapy to the inhibition of specific molecular pathways, in order to reduce tumor burden as typified by chemotherapy and radiation. However, cancer therapy may inherently be a double-edged sword as radiation-induced apoptotic tumor cells can promote tumor growth (the Révész phenomenon) [4, 15, 41-44]. Radiation induces DNA damage that relays a stress signal in cells which activates the mitochondrial pathway of apoptosis [10]. Stimulating cell apoptosis is a well-known mechanism for killing tumor cells, yet dying tumor cells can usurp the apoptotic process to secrete pro-tumorigenesis signals to accelerate repopulation of the radiation-treated tumors $[19,20]$.

The Révész phenomenon has been corroborated in several follow-up studies of radiation-induced cell death [15, 41, 42, 45-47]. Thus, dead and dying tumor cells ("debris") contribute to an underappreciated component of the tumor microenvironment that may promote tumor progression and tumor dormancy escape $[1-3,9,11,14,17,36,48]$. Radiation is a therapy offered to patients with the treatment schedule consisting of daily fractions that are divided off to give time for normal tissues that have been affected by the radiation to recover [7]. It has previously been assumed that most of the dead cells created by radiation would be engulfed through phagocytosis and efferocytosis; thus, tumor recurrence would only proliferate slowly by the minimal tumor cells left behind from the radiation [5]. During the intervals between radiation treatments, surviving tumor cells are stimulated by the apoptotic debris to repopulate at an accelerating rate [7, 49] (Fig. 1a). High expression of pro-apoptotic signals, such as caspase-3, correlates with tumor reoccurrence in patients with postoperative radiotherapy [50]. Unfortunately, radiation therapy may not always be a complete treatment option for cancer patients. Further studies are urgently needed to combine radiation with "pro-resolving" therapies which stimulate the resolution of debris [21].

\section{Chemotherapy-stimulated cancer}

As apoptosis is the body's main mechanism of cell death, most chemotherapies act to induce apoptosis of tumor cells [12]. After chemotherapy or targeted therapy, the dying tumor cells ("debris") should then be engulfed by immune cells, such as macrophages, neutrophils, and dendritic cells. However, when tumor cell debris generated by chemotherapy and a subthreshold inoculum of living tumor cells that would not otherwise proliferate into a tumor are co-injected into an animal model, there is potent stimulation of tumor growth [1-3]. Chemotherapy has the potential to directly promote tumor repopulation and/or metastasis through the production of tumor cell debris-stimulated eicosanoids and cytokines [1, 2, 14] (Fig. 1b). Chemotherapy-generated debris promotes tumorigenesis by stimulating the release of a cytokine storm of pro-inflammatory cytokines such as IL-6 and TNF- $\alpha$ by macrophages $[1,2,14]$ (Fig. 1b). This is similar to the cell-death stimulated eicosanoid-driven cytokine storm in infection such as COVID-19 [51, 52]. Discovering a way to overcome this paradoxical dilemma or Pandora's 


\section{a Radiation-stimulated cancer}

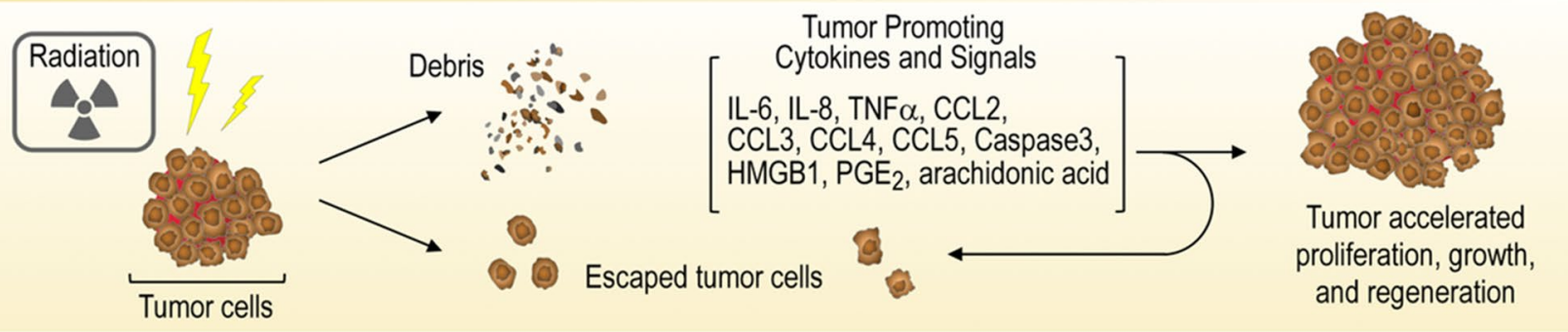

\section{b Chemotherapy-stimulated cancer}

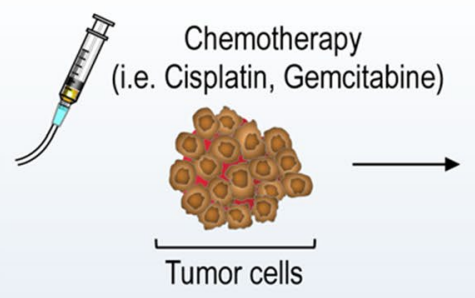

c Surgery-stimulated cancer

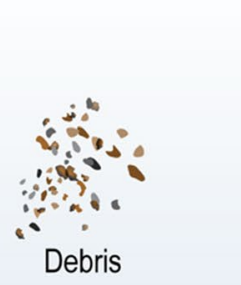

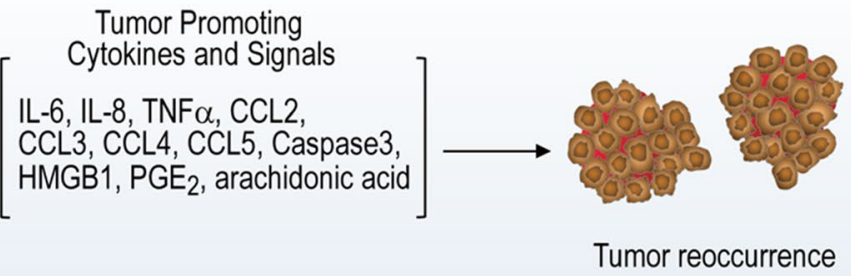

Tumor Promoting

-6, $11-8, \mathrm{TNF}_{\alpha}, \mathrm{CCL} 2$ CCL3, CCL4, CCL5, Caspase3, HMGB1, $\mathrm{PGE}_{2}$, arachidonic acid

Tumor reoccurrence

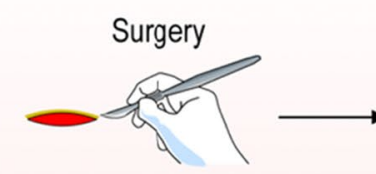

Removal of primary tumor

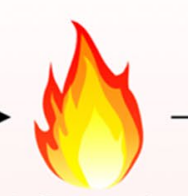

Inflammation

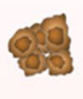

Tumor dormancy escape

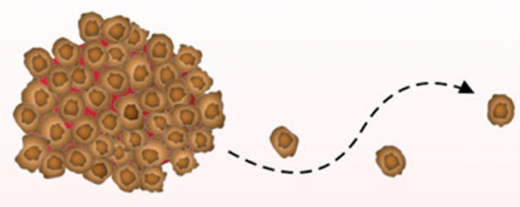

Tumor growth and metastasis
Fig. 1 The general mechanisms for (a) radiation-stimulated cancer, (b) chemotherapy-stimulated cancer, and (c) surgery-stimulated cancer. (a) After stimulating the tumor cells with radiation therapy, debris is created from the dead cells; however, when there are escaped living tumor cells, they are stimulated by the secreted proinflammatory signals from the debris to increase tumor proliferation, growth, and regeneration. (b) Chemotherapy generates debris from

box is crucial in order to prevent tumor reoccurrence after cancer therapy.

Despite the effectiveness of chemotherapy as a frontline cancer therapy, accumulating compelling evidence from animal models suggests that chemotherapy may stimulate tumor growth and metastasis [1, 43, 53-70]. However, the mechanisms for chemotherapy-induced tumor metastasis remain poorly understood. In the subthreshold inoculum model, instead of suppressing tumor growth, chemotherapy promoted tumor growth in small or dormant tumors via tumor-promoting debris [1, 3]. Similarly, chemotherapygenerated tumor cell "debris" (apoptotic and necrotic cells and cell fragments) promotes cancer progression and metastasis via stimulating an eicosanoid-driven cytokine storm of pro-inflammatory and pro-angiogenic mediators $[1,2,14$, $38,39,57,76]$, polarizing tumor-associated macrophages killing the tumor cells, and the debris stimulates the release of protumorigenic cytokines and signals that leads to tumor reoccurrence. (c) Surgery induces systemic inflammation leading to impairment of the resolution of inflammation, increased tumor angiogenesis, and promotion of tumor dormancy escape, which stimulates tumor growth and metastasis

(TAMs) as well as inducing immunosuppression and limiting anti-tumor immunity $[24,77,78]$. Thus, new approaches to stimulate the clearance of tumor cell debris are urgently needed [1, 21, 40, 78-80].

\section{Surgery-stimulated cancer}

Surgery is usually a first-line therapy to eradicate the bulk mass of the tumor. However, similar to chemotherapy and radiation, surgery can be both beneficial and harmful to cancer patients. Surgery, including biopsies, can trigger tumor dormancy escape via multiple mechanisms including impairing tumor-specific immunity, stimulating inflammation, disrupting the resolution of inflammation, and enhancing tumor angiogenesis [1, 2, 4, 74, 81-93]. Additionally, through the 
systemic and local inflammation as well as immunosuppression induced by surgery, metastasis can be stimulated [81, 83], which can promote proliferation and growth of dormant cancer cells at distant anatomical sites [81, 83] (Fig. 1c). Due to a bimodal pattern of tumor recurrence found in earlystage breast and lung cancers, surgery may induce tumor dormancy escape in micrometastatic lesions thus promoting metastasis [81, 94, 95]. Even healthy individuals exhibit microscopic dormant cancer cells that can be activated to proliferate into tumors from noncancer-related surgeries and anesthesia $[81,96]$. Tumor recurrence and metastasis are common causes of death among various cancer types, including pancreatic, breast, liver, colon, and ovarian cancer $[2,3]$. One-third of patients diagnosed with localized breast cancer exhibit metastatic spread to other locations since their initial diagnosis [83, 97, 98]. Breast cancer patients that have their primary tumor removed surgically have a higher risk for tumor relapse 12 to 18 months post-surgery [83, 90]. The link between surgery and the consequent wound-healing response can promote tumor cell proliferation after surgery, specifically the inflammation associated with wound repair $[83,99,100]$. Surgery is the desired and necessary treatment option for many cancer types, so it is important to enhance the beneficial effects of surgery via pro-resolution ("debris-clearing") lipid mediators to decrease the risk of distant metastasis and tumor recurrence [81].

\section{Mechanisms of debris-stimulated tumor growth}

\subsection{Extracellular vesicles}

Apoptotic cells communicate with neighboring cells through several important mechanisms including direct contact, secretion of bioactive molecules into the extracellular matrix, and production of membrane-bound sub-cellular vesicles [10]. Extracellular vesicles (EVs) are a type of membrane-bound sub-cellular vesicle generated by apoptotic cells that vary in size, role, composition, and cargo [101]. EVs can transmit bioactive small molecules, proteins, and nucleic acids [101]. Radiation therapy, chemotherapy, and other cytotoxic therapies enhance the secretion of EVs from dying tumor cells [102]. Apoptotic derived extracellular vesicles (Apo-EVs) can secrete signals to initiate cell-to-cell communication, which aid in signaling surviving tumor cells to undergo tissue repair and accelerated regeneration [101]. The apoptotic tumor cell-derived signals trigger the mechanistic pathways for tumor growth, metastasis, and post-therapeutic relapse [101]. For example, in murine melanoma, EVs promote tumorigenesis through production of growth factors, suppression of anti-tumor immunity, and stimulation of angiogenesis [101, 103]. Another potential mechanism of the pro-tumorigenic activity of EVs is to transfer nutrients such as amino acids, lipids, citrate, and pyruvate to tumor cells to allow growth in a nutrient-deficient environment [101, 104]. In response to stressful conditions, Apo-EVs can also transfer organelles such as mitochondria to other cells [101]. In addition to nutrients and organelles, Apo-EVs, through horizontal transfer, can transfer DNA to neighboring cells, including tumor cells [101]. EVs can also alter the metabolic profile of pancreatic tumor cells [101, 104]. EVs play an important role in the tumor microenvironment by providing critical stress-induced signals to induce proliferation [101]. Further mechanistic studies of debris-stimulated tumor growth will be critical to uncover important targets to inhibit tumor-derived Apo-EVs and lead to novel cancer therapies [101].

\subsection{Exosomal miR-194-5p}

Tumor repopulation is a major cause of treatment failure in cancer patients after chemotherapy and radiation, including a significant increased incidence in pancreatic cancer [46, 102, 105-107]. As previously described, EVs play an important role in intercellular communication and in promoting cancer progression [102]. MicroRNAs (miRNAs) are a type of small non-coding RNA that can be found as vital cargo inside of EVs [102]. Exosomal miR-194-5p derived from radiation-stimulated tumor cell death promotes tumor cell repopulation [102]. After radiation, there is an increase in exosomes released from dead cells, and when the miRNA contained inside was sequenced, a significant increase in miR-194-5p was found [102]. miR-194-5p can downregulate the transcription factor E2F3 to activate cell cycle arrest and repair in the surviving tumor cells [102]. Also, miR194-5p has been shown to act on tumor repopulating cells to facilitate repair from any damage done to the cell from the radiation [102]. In pancreatic ductal adenocarcinoma, miR194-5p can stimulate tumor growth and progression [102, 108]. Fortunately, low-dose aspirin can inhibit tumor proliferation in pancreatic cancer via suppression of the secretion of exosomes from dying tumor cells [102]. Aspirin triggers SPMs including resolvins and lipoxins that can mediate the intricate anti-tumor activity of aspirin [79]. Thus, aspirin and pro-resolution lipid mediators may enhance the anti-tumor activity of radiation via the clearance of tumor-promoting cell debris and the downregulation of exosomal miR-194-5p.

\subsection{Bax, Bak, and Smac}

Pro-apoptotic proteins Bax, Bak, and Smac are potential markers of prognosis in patients with chemotherapytreated metastatic melanoma [12]. Chemotherapy inhibits 
cancer by eliminating tumor cells through apoptosis, and Bax, Bak, and Smac are key apoptosis regulators [12]. When activated, Bax and Bak create pores in the outer membrane of the mitochondria, initiating mitochondrial outer membrane permeabilization (MOMP), which then releases pro-apoptotic factors, such as Smac [11, 12, 109]. Paradoxically, low expression of Bax, Bak, and Smac is correlated with predicting prolonged progression-free survival (PFS) (>12 months) in metastatic melanoma, and high levels of Bax can correlate with poor outcomes [11, 12, 110-114]. Similarly, high expression levels of Bax are associated with an increased risk of relapse in childhood acute lymphoblastic leukemia and poor prognosis in acute myeloid leukemia and non-Hodgkin lymphoma [12, 113-116]. High expression levels of the Bax antagonist Bcl-2 correlate with an improved prognosis in colorectal, breast, glioma, gastric, and nonsmall cell lung cancer [11, 12, 112, 117-121]. Although seemingly counterintuitive, the paradox of debris-stimulated tumors provides new mechanisms of how chemotherapy can stimulate tumor growth and metastasis.

\subsection{HMGB1}

High mobility group box 1 (HMGB1) is released by dying cells treated with radiation therapy or chemotherapy [7]. HMGB1, a damage associated molecular pattern-DAMP, is released from cells undergoing necrosis or uncontrolled cellular and nuclear swelling in response to injury [7, 122, 123]. HMGB1 can stimulate remaining living tumor cells to promote angiogenesis, proliferation, and facilitate metastasis $[7,106]$. This effect is in response to HMGB 1 binding to the receptor for advanced glycation end-products (RAGE) [7]. RAGE then activates downstream pathways ERK and p38, which initiates cell proliferation [7]. In addition to this mechanism, it was found that higher levels of HMGB 1 expression correspond with lower overall survival of cancer patients [7]. When analyzing serum from patients treated with radiation, it was found that there were higher concentrations of HMGB 1 [7]. Under normal conditions, HMGB 1 is located in the nucleus with roles in DNA repair; however, when secreted to the outside of the cell, this protein is associated with cell proliferation, autophagy, inflammation, and immunity $[7,124,125]$. Due to apoptotic cells being programed to hold HMGB1 within the cell, the extracellular levels of HMGB1 must originate from necrotic cells [7]. Radiation combined with a HMGB1 inhibitor may be a treatment option to suppress the cell proliferation of the escaped tumor cells [7].

\subsection{Caspase-3}

Various caspases can induce tumor cell resistance and tumor cell proliferation in response to various anti-cancer therapies [126, 127]. Caspase- 3 is a cysteine protease that rapidly induces apoptotic death at the termination of dying cells' apoptotic mechanism [7, 12, 129]. High levels of the proapoptotic effector caspase- 3 are associated with aggressive disease, large tumor size, poor prognosis, and poor diseasefree survival in patients receiving postoperative radiotherapy $[20,50,101,116]$. Similarly, patients in an early pathology stage of buccal mucosa squamous cell carcinoma that had low co-expression of caspase- 3 and cleaved caspase- 3 were associated with better disease-specific survival [50]. Activation of caspase- 3 has been shown to stimulate cell proliferation in surviving tumor cells after radiation [7, 128]. Caspase- 3 activates downstream effector cytosolic calcium-independent phospholipase $\mathrm{A}_{2}\left(\mathrm{iPLA}_{2}\right)$ to generate arachidonic acid, which is the precursor of the proinflammatory eicosanoid prostaglandin $\mathrm{E}_{2}\left(\mathrm{PGE}_{2}\right)[7,16$, $50,73,130]$. $\mathrm{PGE}_{2}$ critically regulates processes involved in tumor growth, metastasis, inflammation, and cancer stem cell regeneration [73, 131]. The secretion of $\mathrm{PGE}_{2}$ promotes the survival and rapid growth of escaped tumor cells leading to tumor recurrence [131]. In addition to survival and proliferation, $\mathrm{PGE}_{2}$ can decrease the sensitivity of tumor cells to radiation, leading to tumor cell repopulation $[50,132]$. The production of $\mathrm{PGE}_{2}$ by apoptotic tumor cells occurs in bladder cancer, leading to chemoresistance by stimulating cancer stem cell regrowth [132]. Elevated levels of caspase-3 and cleaved caspase- 3 have been observed in various tumor tissues including glioblastoma [133], melanoma [134], acute myelogenous leukemia [135], breast carcinoma [136, 137], non-small cell lung cancer [138], oral squamous cell carcinoma [139], buccal mucosal squamous cell carcinoma [50], gastric cancer, ovarian cancer, cervical cancer, colorectal cancer, and pancreatic ductal carcinoma [20, 140, 141]. In pancreatic cancer, caspase- 3 not only has been linked to tumor recurrence, but is also an indicator of invasiveness [140]. After investigating the immunoreactivity of caspase-3 in tissue from patients with normal pancreatic tissue, pancreatic duct cell carcinoma (PDC), and intraductal papillarymucinous tumor of the pancreas (IPMT), an overexpression of caspase-3 in PDC and invasive IPMT tissues was identified [140]. This suggests that expression of caspase- 3 may contribute to pancreatic cancer invasiveness. In the previously mentioned bladder cancer model, after neutralizing $\mathrm{PGE}_{2}$ signaling, there was a reduction in the emergence of chemoresistance [132]. Ongoing treatment studies are targeting patients that are undergoing chemotherapy or radiation with an inhibitor that would block $\mathrm{PGE}_{2}$ production or an inhibitor of caspase- 3 to enhance the sensitivity of the cancer cells to the treatment $[7,11,20,50,126,132]$. However, 
a caveat is that $\mathrm{PGE}_{2}$ is critical for a lipid-mediator class switching from pro-inflammatory eicosanoids such as leukotrienes and thromboxanes to the specialized pro-resolving mediators including lipoxins [74, 142].

\section{Potential therapies to target debris-stimulated cancer}

\subsection{Platelet activating factor}

Tumor cells that survive during radiation and chemotherapy undergo accelerated repopulation [143]. The stress induced by these therapies stimulates the production of abundant platelet activating factor-like molecules and overexpression of their receptor in tumor cells [44, 143-147]. Platelet activating factor is a soluble lipid mediator that plays an important role in inflammation and tumorigenesis [143, 148, 149]. The G-protein coupled receptor for platelet activating factor can be found in both tumor cells and cells that penetrate tumors $[143,150]$. During cancer therapies, the dying tumor cells release proinflammatory cytokines such as IL-6, IL-8, and TNF- $\alpha$, which then initiate the production of an agonist of platelet activating factor receptor [143, 151-153]. The platelet activating factor receptor induces tumor cell proliferation and production of survival signals and stimulates tumor angiogenesis [143]. After blocking platelet activating factor in experimental models, there was a decrease in tumor size and an increase in animal survival [143]. Antagonists of platelet activating factor reduce tumor repopulation [143]. Thus, a platelet activating factor antagonist, in combination with chemotherapy or radiation, may prevent debris-simulated repopulation of tumor cells [143].

\subsection{Resolution of inflammation}

Inflammation plays an essential role in debris-stimulated cancers, as it promotes tumor cell growth, repair, and repopulation [21]. Debris-stimulated macrophages and other immune cells stimulate inflammation via secretion of proinflammatory, pro-angiogenic, and pro-tumorigenic eicosanoid-driven cytokines $[1,2,17]$. The resolution of inflammation has been discovered by the Serhan laboratory to be an active process regulated by endogenous specialized proresolving mediators (SPMs), which promote the clearance of debris and terminate inflammation [74, 154-156]. Resolvins $(\mathrm{Rv})$ are SPMs that can stimulate the natural debris-clearing process and terminate inflammatory mechanisms [21, 40, 74]. Unlike conventional anti-inflammatories, resolvins are agonists for the resolution of inflammation, are not immunosuppressive, and act at much lower doses [40].

Debris and cell death can induce tumor-promoting inflammation; therefore, the clearance of debris inhibits tumor growth via stimulation of the resolution of inflammation via resolvins $[1,79,81]$ (Fig. 2). In a pancreatic tumor model, resolvins were found to induce tumor regression in combination with chemotherapy [1]; thus, stimulating the resolution of inflammation via resolvins enhances chemotherapy in this model of cancer as well as genetically engineered models of breast and prostate cancer [1]. SPMs can convert macrophages from a pro-inflammatory phenotype into a pro-resolving phenotype, which enhances debris phagocytosis and inhibits further pro-tumorigenic and pro-inflammatory cytokine secretion [1]. D-series resolvin (RvD)1, RvD2, and E-series resolvin (RvE)1 suppressed tumor repopulation in various tumor types

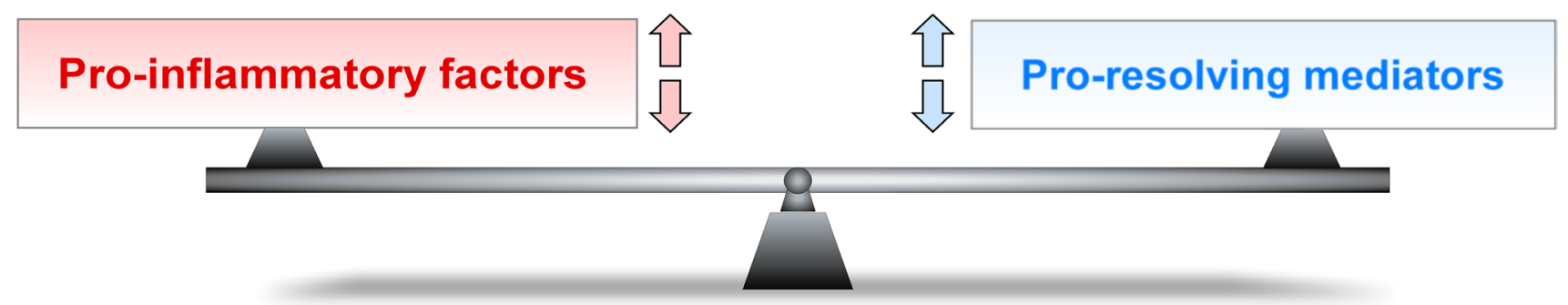

\section{TNF $\alpha$ PGE 2 TGF- $\beta$ CCL8 ADA HGF CXCL-1 MCP-1 IL-6 IL-4 CCL1 CD5 IL-12B CXCL-2 C5a IL-8 IL-7 CCL2 CCL19 CD8A IL-13 CXCL-10 sICAM-1}

Fig. 2 Cancer therapies need to have a balance between pro-inflammatory factors and pro-resolving mediators. Current therapies are focused on the direct killing or removal of tumor cells, which results in an unintentional abundance of proinflammatory/pro-tumorigenesis signals being released from the cancer therapy-generated debris.

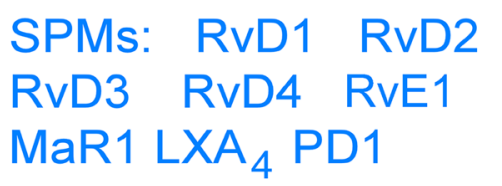

When there are superfluous pro-inflammatory signals, the environment can stimulate tumor recurrence and metastasis, whereas proresolving mediators can stimulate immune cells to engulf the debris and mitigate the pro-tumor signals 
including lung, pancreatic, head and neck cancer, and prostate cancer mouse models [1]. In models of lung cancer and melanoma metastatic, RvD1 and RvD2 were able to suppress metastasis [1]. Resolvins (RvD2, RvD3, and RvD4) were also studied to determine resolvins role in the prevention of tumor dormancy escape and micrometastases from proliferating after surgery [81]. It was found that, if administered before surgery, resolvins suppressed metastasis, increased survival, and inhibited tumor-dormancy escape [81]. Resolvins, in combination with other targeted therapies, can enhance anti-tumor activity and clearance of debris, suppress tumor and metastatic growth, and block further release of pro-inflammatory cytokines [1]. Thus, resolvins are a novel approach to suppress debris-stimulated tumor growth.

In addition to resolvins, omega- 3 fatty acids may also inhibit inflammation in pancreatic cancer $[157,158]$. Targeting soluble epoxide hydrolase inhibition also inhibits debris-stimulated tumor growth in ovarian and liver cancer models via macrophage phagocytosis of debris as well as blocking of pro-inflammatory eicosanoids and cytokines [2, 17]. Although radiation, chemotherapy, and surgery create cell debris, stimulate inflammation, and enhance the tumor microenvironment for repopulation, promoting the resolution of inflammation can stimulate macrophages to engulf debris, block pro-inflammatory factors, and suppress tumor growth and metastasis. Current therapies, such as NSAIDs can be "resolution toxic," meaning they disrupt the endogenous mechanisms of the resolution of inflammation [74, $81,154]$. Thus, agonists of the resolution of inflammation pathway including resolvins represent a promising approach to prevent debris-stimulated tumor growth and accelerated repopulation.

\section{Conclusion}

Chemotherapy, surgery, and radiation all contribute to debris-stimulated cancer. The debris generated from these therapies secrete pro-tumorigenesis factors to promote tumor angiogenesis, proliferation, growth, metastasis, and recurrence. Cancers of all types fall victim to the paradoxical dilemma of debris-stimulated cancer. Fortunately, there are novel approaches to stimulate the resolution or clearance of debris, such as promoting the resolution of inflammation via resolvins or soluble epoxide hydrolase inhibitors, that can be used in combination with traditional cytotoxic cancer therapies to prevent tumor growth and metastasis.
Funding The authors are supported by the Credit Unions Kids at Heart Team (to DP), the C.J. Buckley Pediatric Brain Tumor Fund (DP), and the Joe Andruzzi Foundation (to DP).

\section{Declarations}

Ethics approval Not applicable.

Consent to participate Not applicable.

Conflict of interest The authors declare no competing interests.

\section{References}

1. Sulciner, M. L., et al. (2018). Resolvins suppress tumor growth and enhance cancer therapy. Journal of Experimental Medicine, 215, 115-140.

2. Gartung, A., et al. (2019). Suppression of chemotherapyinduced cytokine/lipid mediator surge and ovarian cancer by a dual COX-2/sEH inhibitor. Proceedings of the National Academy of Sciences of the United States of America, 116, 1698-1703.

3. Chang, J., et al. (2019). Chemotherapy-generated cell debris stimulates colon carcinoma tumor growth via osteopontin. The FASEB Journal, 33, 114-125.

4. Revesz, L. (1956). Effect of tumour cells killed by x-rays upon the growth of admixed viable cells. Nature, 178, 1391-1392.

5. Seelig, K. J., \& Revesz, L. (1960). Effect of lethally damaged tumour cells upon the growth of admixed viable cells in diffusion chambers. British Journal of Cancer, 14, 126-138.

6. van den Brenk, H. A., Crowe, M. C., \& Stone, M. G. (1977). Reactions of the tumour bed to lethally irradiated tumour cells, and the Revesz effect. British Journal of Cancer, 36, 94-104.

7. He, S., et al. (2018). HMGB1 released by irradiated tumor cells promotes living tumor cell proliferation via paracrine effect. Cell Death \& Disease, 9, 648.

8. Green, D. R. (2011). The end and after: How dying cells impact the living organism. Immunity, 35, 441-444.

9. Gregory, C. D., Ford, C. A., \& Voss, J. J. (2016). Microenvironmental effects of cell death in malignant disease. Advances in Experimental Medicine and Biology, 930, 51-88.

10. Gregory, C. D., Paterson, M. (2018) An apoptosis-driven 'oncoregenerative niche': Roles of tumour-associated macrophages and extracellular vesicles. Philosophical Transactions of the Royal Society of London Series B Biological Sciences, 373.

11. Ichim, G., \& Tait, S. W. (2016). A fate worse than death: Apoptosis as an oncogenic process. Nature Reviews Cancer, $16,539-548$.

12. Gutta, C., et al. (2020). Low expression of pro-apoptotic proteins Bax, Bak and Smac indicates prolonged progression-free survival in chemotherapy-treated metastatic melanoma. Cell Death \& Disease, 11, 124 .

13. Hanahan, D., \& Weinberg, R. A. (2000). The hallmarks of cancer. Cell, 100, 57-70.

14. Fishbein, A., et al. (2020). Resolution of eicosanoid/cytokine storm prevents carcinogen and inflammation-initiated hepatocellular cancer progression. Proceedings of the National Academy of Sciences of the United States of America, 117, 21576-21587. 
15. Huang, Q., et al. (2011). Caspase 3-mediated stimulation of tumor cell repopulation during cancer radiotherapy. Nature Medicine, 17, 860-866.

16. Donato, A. L., et al. (2014). Caspase 3 promotes surviving melanoma tumor cell growth after cytotoxic therapy. The Journal of Investigative Dermatology. https://doi.org/10.1038/jid.2014.18

17. Fishbein, A., et al. (2020). Resolution of eicosanoid/cytokine storm prevents carcinogen and inflammation-initiated hepatocellular cancer progression. Proceedings of the National Academy of Sciences of the United States of America. https://doi.org/10. 1073/pnas.2007412117

18. Gregory, C. D., \& Pound, J. D. (2011). Cell death in the neighbourhood: Direct microenvironmental effects of apoptosis in normal and neoplastic tissues. The Journal of Pathology, 223, 177-194.

19. Zhao, R., et al. (2018). Novel roles of apoptotic caspases in tumor repopulation, epigenetic reprogramming, carcinogenesis, and beyond. Cancer and Metastasis Reviews, 37, 227-236.

20. Hu, Q., et al. (2014). Elevated cleaved caspase-3 is associated with shortened overall survival in several cancer types. International Journal of Clinical and Experimental Pathology, 7, 5057-5070.

21. Fishbein, A., Hammock, B. D., Serhan, C. N., \& Panigrahy, D. (2021). Carcinogenesis: Failure of resolution of inflammation? Pharmacology \& Therapeutics, 218, 107670.

22. Ucker, D. S., \& Levine, J. S. (2018). Exploitation of apoptotic regulation in cancer. Frontiers in Immunology, 9, 241.

23. Wyllie, A. H. (1985). The biology of cell death in tumours. Anticancer Research, 5, 131-136.

24. Kornbluth, R. S. (1994). The immunological potential of apoptotic debris produced by tumor cells and during HIV infection. Immunology Letters, 43, 125-132.

25. de Jong, J. S., van Diest, P. J., \& Baak, J. P. (2000). Number of apoptotic cells as a prognostic marker in invasive breast cancer. British Journal of Cancer, 82, 368-373.

26. Sun, B., et al. (2006). Extent, relationship and prognostic significance of apoptosis and cell proliferation in synovial sarcoma. European Journal of Cancer Prevention, 15, 258-265.

27. Ito, Y., et al. (1999). Both cell proliferation and apoptosis significantly predict shortened disease-free survival in hepatocellular carcinoma. British Journal of Cancer, 81, 747-751.

28. McMenamin, M. E., O’Neill, A. J., \& Gaffney, E. F. (1997). Extent of apoptosis in ovarian serous carcinoma: Relation to mitotic and proliferative indices, p53 expression, and survival. Molecular Pathology, 50, 242-246.

29. Hirvikoski, P., et al. (1999). Enhanced apoptosis correlates with poor survival in patients with laryngeal cancer but not with cell proliferation, bcl-2 or p53 expression. European Journal of Cancer, 35, 231-237.

30. Aihara, M., et al. (1995). The frequency of apoptosis correlates with the prognosis of Gleason Grade 3 adenocarcinoma of the prostate. Cancer, 75, 522-529.

31. Leoncini, L., et al. (1993). Correlations between apoptotic and proliferative indices in malignant non-Hodgkin's lymphomas. American Journal of Pathology, 142, 755-763.

32. Naresh, K. N., Lakshminarayanan, K., Pai, S. A., \& Borges, A. M. (2001). Apoptosis index is a predictor of metastatic phenotype in patients with early stage squamous carcinoma of the tongue: A hypothesis to support this paradoxical association. Cancer, 91, 578-584.

33. Jalalinadoushan, M., Peivareh, H., \& Azizzadeh Delshad, A. (2004). Correlation between apoptosis and histological grade of transitional cell carcinoma of urinary bladder. Urology Journal, $1,177-179$.
34. Alcaide, J., et al. (2013). The role and prognostic value of apoptosis in colorectal carcinoma. BMC Clinical Pathology, 13, 24.

35. Holloway, S. B., Colon, G. R., Zheng, W., \& Lea, J. S. (2021). Tumor necrotic debris and high nuclear grade: Newly identified high-risk factors for early-stage endocervical adenocarcinoma. American Journal of Clinical Oncology, 44, 162-168.

36. Connell, P. P., \& Weichselbaum, R. R. (2011). A downside to apoptosis in cancer therapy? Nature Medicine, 17, 780-782.

37. Bonavita, E., Pelly, V. S., \& Zelenay, S. (2018). Resolving the dark side of therapy-driven cancer cell death. Journal of Experimental Medicine, 215, 9-11.

38. Kim, S. H., et al. (2020). Breast cancer cell debris diminishes therapeutic efficacy through heme oxygenase-1-mediated inactivation of M1-like tumor-associated macrophages. Neoplasia, $22,606-616$.

39. Kim, S. H., et al. (2021). Reprograming of tumor-associated macrophages in breast tumor-bearing mice under chemotherapy by targeting heme oxygenase-1. Antioxidants (Basel), 10.

40. Panigrahy, D., Gilligan, M. M., Serhan, C. N., \& Kashfi, K. (2021). Resolution of inflammation: An organizing principle in biology and medicine. Pharmacology \& Therapeutics. https:// doi.org/10.1016/j.pharmthera.2021.107879,107879

41. Chaurio, R., et al. (2013). UVB-irradiated apoptotic cells induce accelerated growth of co-implanted viable tumor cells in immune competent mice. Autoimmunity, 46, 317-322.

42. Ford, C. A., et al. (2015). Oncogenic properties of apoptotic tumor cells in aggressive B cell lymphoma. Current Biology, $25,577-588$

43. Gunjal, P. M., et al. (2015). Evidence for induction of a tumor metastasis-receptive microenvironment for ovarian cancer cells in bone marrow and other organs as an unwanted and underestimated side effect of chemotherapy/radiotherapy. Journal of Ovarian Research, 8, 20.

44. da Silva, I. A., Jr., Chammas, R., Lepique, A. P., \& Jancar, S. (2017). Platelet-activating factor (PAF) receptor as a promising target for cancer cell repopulation after radiotherapy. Oncogenesis, 6, e296.

45. Donato, A. L., et al. (2014). Caspase 3 promotes surviving melanoma tumor cell growth after cytotoxic therapy. The Journal of Investigative Dermatology, 134, 1686-1692.

46. Yu, Y., et al. (2016). eIF4E-phosphorylation-mediated Sox2 upregulation promotes pancreatic tumor cell repopulation after irradiation. Cancer Letters, 375, 31-38.

47. da Silva-Jr, I. A., Chammas, R., Lepique, A. P., \& Jancar, S. (2017). Platelet-activating factor (PAF) receptor as a promising target for cancer cell repopulation after radiotherapy. Oncogenesis, 6, e296.

48. Lauber, K., \& Herrmann, M. (2015). Tumor biology: With a little help from my dying friends. Current Biology, 25, R198-201.

49. Davis, A. J., \& Tannock, J. F. (2000). Repopulation of tumour cells between cycles of chemotherapy: A neglected factor. The lancet Oncology, 1, 86-93.

50. Huang, J. S., et al. (2017). Caspase-3 expression in tumorigenesis and prognosis of buccal mucosa squamous cell carcinoma. Oncotarget, 8, 84237-84247.

51. Panigrahy, D., et al. (2020). Inflammation resolution: A dualpronged approach to averting cytokine storms in COVID-19? Cancer and Metastasis Reviews, 39, 337-340.

52. Hammock, B. D., Wang, W., Gilligan, M. M., \& Panigrahy, D. (2020). Eicosanoids: The overlooked storm in COVID-19? American Journal of Pathology. https://doi.org/10.1016/j.ajpath. 2020.06.010

53. Abubaker, K., et al. (2013). Short-term single treatment of chemotherapy results in the enrichment of ovarian cancer stem 
cell-like cells leading to an increased tumor burden. Molecular Cancer, 12, 24.

54. Volk-Draper, L., et al. (2014). Paclitaxel therapy promotes breast cancer metastasis in a TLR4-dependent manner. Cancer Research, 74, 5421-5434.

55. Chang, Y. S., Jalgaonkar, S. P., Middleton, J. D., \& Hai, T. (2017). Stress-inducible gene Atf3 in the noncancer host cells contributes to chemotherapy-exacerbated breast cancer metastasis. Proceedings of the National Academy of Sciences of the United States of America, 114, E7159-E7168.

56. Karagiannis, G. S., et al. (2017). Neoadjuvant chemotherapy induces breast cancer metastasis through a TMEM-mediated mechanism. Science Translational Medicine, 9.

57. Chang, J., et al. (2018). Chemotherapy-generated cell debris stimulates colon carcinoma tumor growth via osteopontin. The FASEB Journal. https://doi.org/10.1096/fj.201800019RR,fj201 800019RR

58. Li, Q., et al. (2016). Low doses of paclitaxel enhance liver metastasis of breast cancer cells in the mouse model. FEBS Journal, 283, 2836-2852.

59. Liu, G., et al. (2015). Specific chemotherapeutic agents induce metastatic behaviour through stromal- and tumour-derived cytokine and angiogenic factor signalling. The Journal of Pathology, 237, 190-202.

60. de Ruiter, J., Cramer, S. J., Smink, T., \& van Putten, L. M. (1979). The facilitation of tumour growth in the lung by cyclophosphamide in artificial and spontaneous metastases models. European Journal of Cancer, 15, 1139-1149.

61. Orr, F. W., Adamson, I. Y., \& Young, L. (1986). Promotion of pulmonary metastasis in mice by bleomycin-induced endothelial injury. Cancer Research, 46, 891-897.

62. Ormerod, E. J., Everett, C. A., \& Hart, I. R. (1986). Enhanced experimental metastatic capacity of a human tumor line following treatment with 5-azacytidine. Cancer Research, 46, 884-890.

63. Sahu, R. P., et al. (2014). Chemotherapeutic agents subvert tumor immunity by generating agonists of platelet-activating factor. Cancer Research, 74, 7069-7078.

64. Leopold, W. R., Batzinger, R. P., Miller, E. C., Miller, J. A., \& Earhart, R. H. (1981). Mutagenicity, tumorigenicity, and electrophilic reactivity of the stereoisomeric platinum(II) complexes of 1,2-diaminocyclohexane. Cancer Research, 41, 4368-4377.

65. Ramani, V. C., \& Sanderson, R. D. (2014). Chemotherapy stimulates syndecan-1 shedding: A potentially negative effect of treatment that may promote tumor relapse. Matrix Biology, 35, 215-222.

66. Keklikoglou, I., et al. (2019). Chemotherapy elicits pro-metastatic extracellular vesicles in breast cancer models. Nature Cell Biology, 21, 190-202.

67. Chen, X., et al. (2021). DCBLD2 mediates epithelial-mesenchymal transition-induced metastasis by cisplatin in lung adenocarcinoma. Cancers (Basel), 13.

68. Wills, C. A., et al. (2021). Chemotherapy-induced upregulation of small extracellular vesicle-associated PTX3 accelerates breast cancer metastasis. Cancer Research, 81, 452-463.

69. Zhu, X., et al. (2019). Neoadjuvant chemotherapy plays an adverse role in the prognosis of grade 2 breast cancer. Journal of Cancer, 10, 5661-5670.

70. Andrade, L. N. S., et al. (2019). Extracellular vesicles shedding promotes melanoma growth in response to chemotherapy. Science and Reports, 9, 14482.

71. Sulciner, M. L., Gartung, A., Gilligan, M. M., Serhan, C. N., \& Panigrahy, D. (2018). Targeting lipid mediators in cancer biology. Cancer and Metastasis Reviews, 37, 557-572.
72. Greene, E. R., Huang, S., Serhan, C. N., \& Panigrahy, D. (2011). Regulation of inflammation in cancer by eicosanoids. Prostaglandins \& Other Lipid Mediators, 96, 27-36.

73. Wang, D., \& Dubois, R. N. (2010). Eicosanoids and cancer. Nature Reviews Cancer, 10, 181-193.

74. Serhan, C. N. (2014). Pro-resolving lipid mediators are leads for resolution physiology. Nature, 510, 92-101.

75. Serhan, C. N., Hamberg, M., \& Samuelsson, B. (1984). Lipoxins: Novel series of biologically active compounds formed from arachidonic acid in human leukocytes. Proceedings of the National Academy of Sciences of the United States of America, 81, 5335-5339.

76. Chen, Q., et al. (2019). Tumour cell-derived debris and IgG synergistically promote metastasis of pancreatic cancer by inducing inflammation via tumour-associated macrophages. British Journal of Cancer, 121, 786-795.

77. Sekar, D., Hahn, C., Brune, B., Roberts, E., \& Weigert, A. (2012). Apoptotic tumor cells induce IL-27 release from human DCs to activate Treg cells that express CD69 and attenuate cytotoxicity. European Journal of Immunology, 42, 1585-1598.

78. Lavy, M., Gauttier, V., Poirier, N., Barille-Nion, S., \& Blanquart, C. (2021). Specialized pro-resolving mediators mitigate cancer-related inflammation: Role of tumor-associated macrophages and therapeutic opportunities. Frontiers in Immunology, 12, 702785 .

79. Gilligan, M. M., et al. (2019). Aspirin-triggered proresolving mediators stimulate resolution in cancer. Proceedings of the National Academy of Sciences of the United States of America, 116, 6292-6297.

80. Ye, Y., et al. (2018). Anti-cancer and analgesic effects of resolvin D2 in oral squamous cell carcinoma. Neuropharmacology, 139, 182-193.

81. Panigrahy, D., et al. (2019). Preoperative stimulation of resolution and inflammation blockade eradicates micrometastases. The Journal of Clinical Investigation, 129, 2964-2979.

82. Fisher, B., \& Fisher, E. R. (1959). Experimental evidence in support of the dormant tumor cell. Science, 130, 918-919.

83. Krall, J. A., et al., (2018) The systemic response to surgery triggers the outgrowth of distant immune-controlled tumors in mouse models of dormancy. Science Translational Medicine, 10.

84. Ananth, A. A., et al. (2016). Surgical stress abrogates pre-existing protective $\mathrm{T}$ cell mediated anti-tumor immunity leading to postoperative cancer recurrence. PLoS One, 11, e0155947.

85. Lee, J. W., et al. (2009). Surgical stress promotes tumor growth in ovarian carcinoma. Clinical Cancer Research, 15, 2695-2702.

86. Antonio, N., et al. (2015). The wound inflammatory response exacerbates growth of pre-neoplastic cells and progression to cancer. EMBO Journal, 34, 2219-2236.

87. Mathenge, E. G., et al. (2014). Core needle biopsy of breast cancer tumors increases distant metastases in a mouse model. Neoplasia, 16, 950-960.

88. Alieva, M., et al. (2017). Preventing inflammation inhibits biopsy-mediated changes in tumor cell behavior. Science and Reports, 7, 7529.

89. Hobson, J., et al. (2013). Acute inflammation induced by the biopsy of mouse mammary tumors promotes the development of metastasis. Breast Cancer Research and Treatment, 139, 391-401.

90. Retsky, M., Demicheli, R., Hrushesky, W., Baum, M., \& Gukas, I. (2010). Surgery triggers outgrowth of latent distant disease in breast cancer: An inconvenient truth? Cancers (Basel), 2, 305-337.

91. Cata, J. P., et al. (2017). Inflammation and pro-resolution inflammation after hepatobiliary surgery. World Journal of Surgical Oncology, 15, 152. 
92. Shakhar, G., \& Ben-Eliyahu, S. (2003). Potential prophylactic measures against postoperative immunosuppression: Could they reduce recurrence rates in oncological patients? Annals of Surgical Oncology, 10, 972-992.

93 Forget, P., Simonet, O., \& De Kock, M. (2013). Cancer surgery induces inflammation, immunosuppression and neo-angiogenesis, but is it influenced by analgesics? F1000Res, 2, 102.

94. Demicheli, R., et al. (1994). Local recurrences following mastectomy: Support for the concept of tumor dormancy. Journal of the National Cancer Institute, 86, 45-48.

95. Retsky, M. W., Demicheli, R., Hrushesky, W. J., Baum, M., \& Gukas, I. D. (2008). Dormancy and surgery-driven escape from dormancy help explain some clinical features of breast cancer. APMIS, 116, 730-741.

96. Bovill, J. G. (2010). Surgery for cancer: Does anesthesia matter? Anesthesia and Analgesia, 110, 1524-1526.

97. Pantel, K., Brakenhoff, R. H., \& Brandt, B. (2008). Detection, clinical relevance and specific biological properties of disseminating tumour cells. Nature Reviews Cancer, 8, 329-340.

98. Husemann, Y., et al. (2008). Systemic spread is an early step in breast cancer. Cancer Cell, 13, 58-68.

99. Dillekas, H., et al. (2016). The recurrence pattern following delayed breast reconstruction after mastectomy for breast cancer suggests a systemic effect of surgery on occult dormant micrometastases. Breast Cancer Research and Treatment, 158, 169-178.

100. Dillekas, H., Transeth, M., Pilskog, M., Assmus, J., \& Straume, O. (2014). Differences in metastatic patterns in relation to time between primary surgery and first relapse from breast cancer suggest synchronized growth of dormant micrometastases. Breast Cancer Research and Treatment, 146, 627-636.

101. Gregory, C. D., \& Dransfield, I. (2018). Apoptotic tumor cellderived extracellular vesicles as important regulators of the oncoregenerative niche. Frontiers in Immunology, 9, 1111.

102. Jiang, M. J., et al. (2020). Dying tumor cell-derived exosomal miR-194-5p potentiates survival and repopulation of tumor repopulating cells upon radiotherapy in pancreatic cancer. Molecular Cancer, 19, 68.

103. Lin, L. Y., et al. (2016). Tumour cell-derived exosomes endow mesenchymal stromal cells with tumour-promotion capabilities. Oncogene, 35, 6038-6042.

104 Zhao, H., et al. (2016). Tumor microenvironment derived exosomes pleiotropically modulate cancer cell metabolism. Elife, 5, e10250.

105. Kim, J. J., \& Tannock, I. F. (2005). Repopulation of cancer cells during therapy: An important cause of treatment failure. Nature Reviews Cancer, 5, 516-525.

106. Fang, C., et al. (2018). microRNA-193a stimulates pancreatic cancer cell repopulation and metastasis through modulating TGF-beta2/TGF-betaRIII signalings. Journal of Experimental \& Clinical Cancer Research, 37, 25.

107. Cheng, J., et al. (2015). Dying tumor cells stimulate proliferation of living tumor cells via caspase-dependent protein kinase Cdelta activation in pancreatic ductal adenocarcinoma. Molecular Oncology, 9, 105-114.

108. Zhang, J., et al. (2014). Upregulation of miR-194 contributes to tumor growth and progression in pancreatic ductal adenocarcinoma. Oncology Reports, 31, 1157-1164.

109. Tait, S. W., \& Green, D. R. (2010). Mitochondria and cell death: Outer membrane permeabilization and beyond. Nature Reviews Molecular Cell Biology, 11, 621-632.

110. Dawson, S. J., et al. (2010). BCL2 in breast cancer: A favourable prognostic marker across molecular subtypes and independent of adjuvant therapy received. British Journal of Cancer, 103, $668-675$.
111. Anagnostou, V. K., et al. (2010). High expression of BCL-2 predicts favorable outcome in non-small cell lung cancer patients with non squamous histology. BMC Cancer, 10, 186.

112. Renouf, D. J., et al. (2009). BCL-2 expression is prognostic for improved survival in non-small cell lung cancer. Journal of Thoracic Oncology, 4, 486-491.

113. Hogarth, L. A., \& Hall, A. G. (1999). Increased BAX expression is associated with an increased risk of relapse in childhood acute lymphocytic leukemia. Blood, 93, 2671-2678.

114. Kaparou, M., et al. (2013). Enhanced levels of the apoptotic BAX/BCL-2 ratio in children with acute lymphoblastic leukemia and high-risk features. Genetics and Molecular Biology, 36, $7-11$.

115. Kohler, T., et al. (2002). High Bad and Bax mRNA expression correlate with negative outcome in acute myeloid leukemia (AML). Leukemia, 16, 22-29.

116. Bairey, O., Zimra, Y., Shaklai, M., Okon, E., \& Rabizadeh, E. (1999). Bcl-2, Bcl-X, Bax, and Bak expression in short- and long-lived patients with diffuse large B-cell lymphomas. Clinical Cancer Research, 5, 2860-2866.

117. Meterissian, S. H., et al. (2001). Bcl-2 is a useful prognostic marker in Dukes' B colon cancer. Annals of Surgical Oncology, $8,533-537$

118. Vargas-Roig, L. M., et al. (2008). Prognostic value of Bcl-2 in breast cancer patients treated with neoadjuvant anthracycline based chemotherapy. Molecular Oncology, 2, 102-111.

119. McDonald, F. E., et al. (2002). The prognostic influence of bcl-2 in malignant glioma. British Journal of Cancer, 86, 1899-1904.

120. Inada, T., Kikuyama, S., Ichikawa, A., Igarashi, S., \& Ogata, Y. (1998). Bcl-2 expression as a prognostic factor of survival of gastric carcinoma. Anticancer Research, 18, 2003-2010.

121. Labi, V., \& Erlacher, M. (2015). How cell death shapes cancer. Cell Death \& Disease, 6, e1675.

122. Balkwill, F. (2009). Tumour necrosis factor and cancer. Nature Reviews Cancer, 9, 361-371.

123. Pisetsky, D. (2011). Cell death in the pathogenesis of immunemediated diseases: The role of HMGB1 and DAMP-PAMP complexes. Swiss Medical Weekly, 141, w13256.

124. Kang, R., et al. (2017). Intracellular HMGB1 as a novel tumor suppressor of pancreatic cancer. Cell Research, 27, 916-932.

125. Bertheloot, D., \& Latz, E. (2017). HMGB1, IL-1alpha, IL-33 and S100 proteins: Dual-function alarmins. Cellular \& Molecular Immunology, 14, 43-64.

126. Liu, P. F., et al. (2017). Expression levels of cleaved caspase-3 and caspase- 3 in tumorigenesis and prognosis of oral tongue squamous cell carcinoma. PLoS One, 12, e0180620.

127. Coutinho-Camillo, C. M., Lourenco, S. V., Nishimoto, I. N., Kowalski, L. P., \& Soares, F. A. (2011). Caspase expression in oral squamous cell carcinoma. Head and Neck, 33, 1191-1198.

128. Zhao, X., et al. (2006). Caspase-3-dependent activation of calcium-independent phospholipase A2 enhances cell migration in non-apoptotic ovarian cancer cells. Journal of Biological Chemistry, 281, 29357-29368.

129. Hellwig, C. T., Passante, E., \& Rehm, M. (2011). The molecular machinery regulating apoptosis signal transduction and its implication in human physiology and pathophysiologies. Current Molecular Medicine, 11, 31-47.

130. Nakanishi, M., \& Rosenberg, D. W. (2013). Multifaceted roles of PGE2 in inflammation and cancer. Semin Immunopathol, 35, 123-137.

131. Wang, D., \& DuBois, R. N. (2018). Role of prostanoids in gastrointestinal cancer. The Journal of Clinical Investigation, 128, 2732-2742.

132. Kurtova, A. V., et al. (2015). Blocking PGE2-induced tumour repopulation abrogates bladder cancer chemoresistance. Nature, 517, 209-213. 
133. Gdynia, G., et al. (2007). Basal caspase activity promotes migration and invasiveness in glioblastoma cells. Molecular Cancer Research, 5, 1232-1240.

134. Liu, Y. R., et al. (2013). Basal caspase-3 activity promotes migration, invasion, and vasculogenic mimicry formation of melanoma cells. Melanoma Research, 23, 243-253.

135. Estrov, Z., et al. (1998). Caspase 2 and caspase 3 protein levels as predictors of survival in acute myelogenous leukemia. Blood, 92, 3090-3097.

136. Vakkala, M., Paakko, P., \& Soini, Y. (1999). Expression of caspases 3, 6 and 8 is increased in parallel with apoptosis and histological aggressiveness of the breast lesion. British Journal of Cancer, 81, 592-599.

137. O'Donovan, N., et al. (2003). Caspase 3 in breast cancer. Clinical Cancer Research, 9, 738-742.

138. Tormanen-Napankangas, U., Soini, Y., Kahlos, K., Kinnula, V., \& Paakko, P. (2001). Expression of caspases-3, -6 and -8 and their relation to apoptosis in non-small cell lung carcinoma. International Journal of Cancer, 93, 192-198.

139. Hague, A., et al. (2004). Caspase-3 expression is reduced, in the absence of cleavage, in terminally differentiated normal oral epithelium but is increased in oral squamous cell carcinomas and correlates with tumour stage. The Journal of Pathology, 204, 175-182.

140. Satoh, K., Kaneko, K., Hirota, M., Toyota, T., \& Shimosegawa, T. (2000). The pattern of CPP32/caspase-3 expression reflects the biological behavior of the human pancreatic duct cell tumors. Pancreas, 21, 352-357.

141. Jakubowska, K., et al. (2016). Reduced expression of caspase-8 and cleaved caspase- 3 in pancreatic ductal adenocarcinoma cells. Oncology Letters, 11, 1879-1884.

142. Levy, B. D., Clish, C. B., Schmidt, B., Gronert, K., \& Serhan, C. N. (2001). Lipid mediator class switching during acute inflammation: Signals in resolution. Nature Immunology, 2, 612-619.

143. da Silva Junior, I. A., de Sousa Andrade, L. N., Jancar, S., \& Chammas, R. (2018). Platelet activating factor receptor antagonists improve the efficacy of experimental chemo- and radiotherapy. Clinics (Sao Paulo), 73, e792s.

144. de Oliveira, S. I., et al. (2010). Platelet-activating factor receptor (PAF-R)-dependent pathways control tumour growth and tumour response to chemotherapy. BMC Cancer, 10, 200.

145. Li, T., et al. (2003). The epidermal platelet-activating factor receptor augments chemotherapy-induced apoptosis in human carcinoma cell lines. Journal of Biological Chemistry, 278, 16614-16621.

146. Yao, B., et al. (2017). PAFR selectively mediates radioresistance and irradiation-induced autophagy suppression in prostate cancer cells. Oncotarget, 8, 13846-13854.
147. Sahu, R. P., et al. (2016). Radiation therapy generates plateletactivating factor agonists. Oncotarget, 7, 20788-20800.

148. Melnikova, V., \& Bar-Eli, M. (2007). Inflammation and melanoma growth and metastasis: The role of platelet-activating factor (PAF) and its receptor. Cancer and Metastasis Reviews, 26, 359-371.

149. Jancar, S., \& Chammas, R. (2014). PAF receptor and tumor growth. Current Drug Targets, 15, 982-987.

150. Sugimoto, T., et al. (1992). Molecular cloning and characterization of the platelet-activating factor receptor gene expressed in the human heart. Biochemical and Biophysical Research Communications, 189, 617-624.

151. Walterscheid, J. P., Ullrich, S. E., \& Nghiem, D. X. (2002). Platelet-activating factor, a molecular sensor for cellular damage, activates systemic immune suppression. Journal of Experimental Medicine, 195, 171-179.

152. Sahu, R. P., et al. (2012). The environmental stressor ultraviolet B radiation inhibits murine antitumor immunity through its ability to generate platelet-activating factor agonists. Carcinogenesis, $33,1360-1367$.

153. Yao, Y., et al. (2009). Ultraviolet B radiation generated plateletactivating factor receptor agonist formation involves EGF-Rmediated reactive oxygen species. The Journal of Immunology, 182, 2842-2848.

154. Serhan, C. N., \& Levy, B. D. (2018). Resolvins in inflammation: Emergence of the pro-resolving superfamily of mediators. The Journal of Clinical Investigation, 128, 2657-2669.

155. Serhan, C. N., et al. (2002). Resolvins: A family of bioactive products of omega-3 fatty acid transformation circuits initiated by aspirin treatment that counter proinflammation signals. Journal of Experimental Medicine, 196, 1025-1037.

156. Serhan, C. N., et al. (2000). Novel functional sets of lipidderived mediators with antiinflammatory actions generated from omega-3 fatty acids via cyclooxygenase 2 -nonsteroidal antiinflammatory drugs and transcellular processing. Journal of Experimental Medicine, 192, 1197-1204.

157. Janakiram, N. B., Mohammed, A., \& Rao, C. V. (2011). Role of lipoxins, resolvins, and other bioactive lipids in colon and pancreatic cancer. Cancer and Metastasis Reviews, 30, 507-523.

158. Nabavi, S. F., et al. (2015). Omega-3 polyunsaturated fatty acids and cancer: Lessons learned from clinical trials. Cancer and Metastasis Reviews, 34, 359-380.

Publisher's note Springer Nature remains neutral with regard to jurisdictional claims in published maps and institutional affiliations. 